
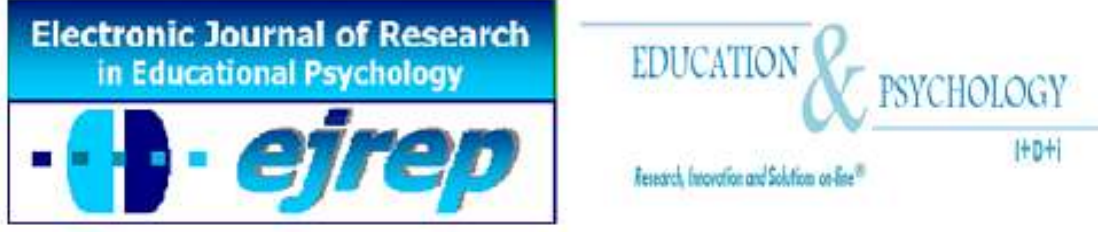

\title{
Burnout y Desarrollo de Competencias en la Formación Inicial de Maestros
}

\author{
Antonio J. Rodríguez-Hidalgo ${ }^{1}$, Juan Calmaestra ${ }^{2}$ \\ e Irene Dios ${ }^{1}$
}

\author{
${ }^{1}$ Departamento de Psicología, Universidad de Córdoba, Córdoba \\ ${ }^{2}$ Departamento Interfacultativo de Psicología Evolutiva y de la Educación, \\ Universidad Autónoma de Madrid, Madrid
}

España

Correspondencia: Antonio Jesús Rodríguez Hidalgo. Facultad de Ciencias de la Educación, Avda. San Alberto Magno, S/N, 14071, Córdoba. España. E-mail: ajrodriguez@uco.es

(C) Education \& Psychology I+D+i and Editorial EOS (Spain) 


\section{Resumen}

Introducción. El síndrome de burnout afecta negativamente al rendimiento académico. Se somete a estudio la relación entre burnout académico y la auto-percepción del desarrollo de competencias durante la formación inicial de maestros.

Método. Una muestra de 274 estudiantes (edad media = 20.61 años) de los Grados de Educación Infantil y Primaria participaron cumplimentando un cuestionario ad hoc sobre autopercepción de competencias y la escala de burnout MBI-SS. El cuestionario se administró anónima, voluntaria e individualmente. Se comparan puntuaciones medias obtenidas en ambos cuestionarios a través de T de Students y ANOVA.

Resultados. El grupo con alto nivel de burnout presenta niveles más bajos de auto-percepción en el desarrollo competencias. Solo una dimensión del burnout, eficacia, se asociaba positivamente con la auto-percepción del desarrollo de competencias.

Discusión y conclusiones. Se infiere la necesidad de realizar un trabajo formativo que estimule el desarrollo y la auto-percepción de las competencias personales y sistémico/contextuales.

Palabras Clave: Burnout, competencias, formación inicial, maestro, eficacia. 


\title{
Burnout and development of competences in initial teacher training
}

\begin{abstract}
Introduction. The burnout syndrome negatively affects the students' academic performance. The relation between academic burnout and the self-perception of skills in initial teacher training is subjected to analysis.

Method. A sample of 274 students (average age= 20,61 years old) from the Bachelor Degree in Early Childhood Education and the Bachelor Degree in Primary Education were involved in the study by filling in the Skills development in the Teaching Training programme questionnaire and the burnout MBI-SS scale. The questionnaire was administered anonymously, voluntarily and individually. Mean scores on both questionnaires through $\mathrm{T}$ Students and ANOVA were compared.
\end{abstract}

Results. The group of students with a higher burnout level presented lower levels of selfperception in the development of their skills. Only one dimension of burnout, efficacy, was positively associated with self-perception of the skills' development.

Discussion and Conclusion. It becomes clear the need to carry out a training work that stimulates the acquisition and self-perception of personal and systemic-contextual skills.

Keywords: Burnout, skills, initial training, teacher, efficacy. 


\section{Introducción}

La formación universitaria, en los últimos años, orienta el rendimiento de los egresados hacia el desarrollo de competencias. Se han realizado distintas investigaciones que concluyen con la existencia de diversos factores que pueden incidir negativamente en el rendimiento de estudiantes universitarios. Entre estos factores destacan el estrés estudiantil (Caballero, Hederich y Palacio, 2010) y una de sus consecuencias, el burnout, debido a las actividades académicas formativas (González y Landero, 2007), que condicionan negativamente los procesos de enseñanza-aprendizaje y, por ende, el rendimiento académico. Sin embargo esta línea de estudios, que relaciona el estrés y el burnout estudiantil con el rendimiento académico, no ha contemplado este último concebido como desarrollo competencial.

Es otra línea diferente de estudios la que en los últimos años ha realizado investigaciones sobre el desarrollo de competencias. Esta línea se ha orientado más hacia el estudio de los perfiles competenciales y su desarrollo (Castejón, Cantero y Pérez, 2008), tanto de los preprofesionales en la formación inicial de nivel universitario (p. ej.: Sáiz y Román, 2011; Villardón-Gallego, Yániz, Achurra, Iraurgi y Aguilar, 2013), como de los docentes graduados en activo (p. ej.: Pérez-Escoda, Filella, Alegre y Bisquerra, 2012; Valdivieso, Carbonero y Martín-Antón, 2013). Algunas de estas investigaciones concluyen con que la autoevaluación de las competencias es el procedimiento apropiado para la evaluación del desempeño del sujeto (Pena, Rey y Extremera, 2012; Valdivieso et al., 2013).

Habida cuenta del vacío existente en la literatura científica de investigaciones sobre las posibles relaciones entre burnout académico y desarrollo de competencias en estudiantes universitarios, el presente estudio pone el foco en estas posibles relaciones con la pretensión de avanzar en su conocimiento.

\section{El burnout académico}

El burnout de estudiantes en su actividad académica comenzó a investigarse siguiendo el modelo teórico sobre burnout profesional de estudios anteriores (Gil-Monte y Peiró, 1999; Schaufeli, Leiter, Maslach y Jackson, 1996; Maslach y Jackson, 1981), en algunos casos, concretamente en los propios docentes de infantil y primaria en ejercicio (Arís, 2009). El burnout académico es concebido como sentirse agotado debido a las demandas del estudio, poseer una actitud crítica e indiferente hacia el estudio y sentirse incompetente como estudiante (Schau- 
feli, Martínez, Marques, Salanova y Bakker, 2002; Schaufeli, Salanova, González-Romá, y Bakker, 2002). El constructo burnout académico está constituido por tres dimensiones agotamiento, cinismo y eficacia- como se refleja en los factores tenidos en cuenta para la medición del mismo en el instrumento Maslach Burnout Inventory-Student Survey (MBI-SS).

En los últimos años, varias investigaciones abordan el estudio del burnout académico en muestras pre-ocupacionales utilizando la escala MBI-SS (Bresó, Salanova y Schaufeli, 2007; Fontes de Oliva, Andrade, Rodrigues de Abreu, Vieira y Matos, 2012; Salanova, Martínez, Bresó, Llorens y Grau, 2005; Schaufeli, Salanova et al., 2002), contemplándose como útil para detectar de forma precoz indicios característicos de dicho síndrome y poder promover intervenciones tempranas que orienten hacia el éxito académico.

Un estudiante está más “quemado" cuando mediante la escala MBI-SS manifiesta altas puntuaciones en agotamiento y cinismo, y bajas puntuaciones en eficacia (Salanova, Martínez et al. 2005; Schaufeli, Martínez et al., 2002). La eficacia ha sido considerada por algunos estudios como fundamental en el proceso del burnout, explicando que este fenómeno puede considerarse como la crisis de la eficacia (Salanova, Bresó y Schaufeli, 2005; Salanova, Cifre, Grau, Llorens y Martínez, 2005) y que la eficacia es un predictor importante del burnout académico (Durán, Extremera, Rey, Fernández-Berrocal y Montalbán, 2006; Salanova, Martínez y Llorens, 2012; Schwarzer y Hallum, 2008). Existe por tanto una relación negativa entre la dimensión de eficacia percibida y el burnout.

En la última década, algunas investigaciones han estudiado la relación entre burnout académico en estudiantes y otras variables como el rendimiento académico (Salanova, Martínez et al., 2005), el desempeño, contemplado como expectativas de éxito (Manzano, 2002), y el desarrollo profesional (Martínez y Salanova, 2003). Son destacables los hallazgos de Salanova, Martínez et al. (2005) que detectaron relaciones significativas entre bienestar psicológico y rendimiento académico, observando que a mayor rendimiento académico, menor agotamiento, mayor eficacia, vigor, dedicación, autoeficacia, felicidad y satisfacción relacionada con los estudios. Sin embargo, muy poco se sabe sobre la relación entre el burnout académico de estudiantes y la adquisición y desarrollo de las competencias que los titulados universitarios deben tener al finalizar sus estudios. 


\section{Autoeficacia en estudiantes universitarios}

Desde que Bandura (1977) introdujo en la década de los setenta el concepto de autoeficacia, son cada vez más las investigaciones que han estudiado el papel que juega la autoeficacia en el desarrollo académico y personal del estudiante (Bandura, 2012; Cho y Serena, 2013; González, Donolo, Rinaudo y Paolini, 2011). En la Teoría Socio-Cognitiva, la autoeficacia es entendida como la "creencia en las capacidades propias del individuo para organizar y ejecutar cursos de acción necesarios para alcanzar determinados objetivos" (Bandura, 1997, pág. 3), afectando a su comportamiento, motivación y perseverancia en el logro de dichos objetivos (Hultell, Melin y Gustavsson, 2013). La auto-percepción sobre las capacidades, entre otros factores, afecta al procesamiento cognitivo de la información sobre el propio desempeño, y conocer estos efectos nos permite comprender las condiciones bajo las que las personas sacan el máximo de sus experiencias de dominio (Salanova et al., 2012). La autoeficacia en estudiantes universitarios es concebida como la seguridad y la confianza que dichos estudiantes tienen en sus habilidades para afrontar y desarrollar las tareas y actividades académicas que le son encomendadas (García-Ros y Pérez-González, 2011).

Los cambios en la autoeficacia están fuertemente unidos a los cambios en los estados de bienestar, como son el burnout y el engagement. Cuando el estudiante experimenta pensamientos negativos acerca de las capacidades que posee, puede desarrollar una percepción más baja de dichas capacidades, que a su vez podría reforzar la probabilidad de la aparición del rendimiento insuficiente que teme (Bresó, Schaufeli, y Salanova, 2011). Sin embargo, cuando se hace una auto-observación positiva de las propias competencias, la persona promueve la aparición de un mecanismo auto-motivador que le posibilita alcanzar niveles más altos de autoeficacia (Caballero et al. 2010).

Numerosos estudios avalan la importancia de los recursos personales para manejar y hacer frente a las demandas internas y externas en situaciones de estrés (Cho y Serena, 2013; Durán et al., 2006). La persona que siente que no tiene ninguna posibilidad de alcanzar su meta no invertirá recursos para ello, ni tampoco se invertirán demasiados recursos cuando una meta es fácilmente alcanzable con una mínima inversión (Beck y Schmidt, 2012). Malinen et al. (2013) añaden que los individuos que experimentan el éxito fácil solo esperan resultados rápidos y pronto se desanimarán ante cualquier dificultad. Por otra parte, las personas con una mayor autoeficacia, suelen invertir más recursos (Beck y Schmidt, 2012), invierten más esfuerzo, tiempo, persistencia, se recuperan rápidamente ante los contratiempos y mantienen el 
compromiso con sus objetivos (Schwarzer y Hallum, 2008) logrando mayor rendimiento que personas con menor autoeficacia.

\section{Desarrollo de competencias en estudiantes universitarios}

En los últimos años es emergente la investigación sobre el desarrollo de las competencias en estudiantes universitarios (Beneitone et al., 2007; de la Fuente, Justicia, Casanova y Trianes, 2005; Palmer, Montaño y Palou, 2009; Pertegal-Felices, Castejón-Costa y Martínez, 2011; Shen-Miller, 2012), considerando la idea de que los recursos que se obtienen en esta etapa servirán como base para la formación permanente, en este caso, del profesorado (Palomera, Fernández-Berrocal y Brackett, 2008). Caballero et al. (2010) afirman que muchos de los estudiantes logran desarrollar las competencias correctamente, pero otros presentan grandes dificultades en la formación superior que pueden conducir a la "mortalidad académica". Según estos autores, la carga de estrés sufrida por los mismos, en ausencia de las estrategias adecuadas para afrontar las exigencias académicas, podría desembocar en sentimientos de incompetencia. Del mismo modo podría provocar comportamientos de escape como forma de afrontamiento inapropiada para la solución del conflicto (Gustems y Calderón, 2014). En este sentido Martínez y Salanova (2003) afirman que la falta de competencia y eficacia, la aparición de sentimientos negativos de inadecuación y la disminución de expectativas personales podrían conducir a una autoevaluación negativa y a la aparición de sentimientos de fracaso y baja autoestima.

Los actuales planes de estudios universitarios de grado en el EEES están orientados a la consecución de varios tipos de competencias. Algunas de las más importantes se agrupan bajo las nominaciones de genéricas/transversales y específicas (ANECA, 2005; González y Wagenaar, 2003). Las competencias genéricas o transversales son comunes a todas las carreras, por lo que todo estudiante universitario debe adquirirlas con independencia de la titulación que curse (ANECA, 2005). Entre las competencias genéricas o transversales se encuentran las de tipo instrumental, personal y sistémico (González y Wagenaar, 2003). Por su parte, las competencias específicas son propias y características de cada carrera universitaria. En el caso de la profesión de maestro sus competencias específicas se describen en el Libro Blanco de Magisterio (ANECA, 2005). Estas competencias específicas son comunes a todos los maestros, con independencia de su especialidad o mención. 


\section{Objetivos e hipótesis}

Contemplando la revisión de la literatura científica sobre burnout académico en estudiantes y la orientación formativa de la Educación Superior hacia el desarrollo de competencias transversales y específicas, se cuestiona qué relación habrá entre estos aspectos. Para ello, se estudia la autopercepción de los estudiantes sobre el desarrollo de sus competencias trasversales y específicas, se miden sus niveles de burnout académico y se estudian las posibles diferencias en su autopercepción sobre el desarrollo de sus competencias en función de su nivel de burnout. Se espera que el alumnado que presente un alto nivel del síndrome de burnout presentará una autopercepción inferior del grado de desarrollo de sus competencias transversales y específicas frente al alumnado cuyo nivel de burnout sea inferior.

\section{Método}

\section{Participantes}

La población de partida estaba compuesta por 437 estudiantes matriculados en segundo curso de los grados de Educación Infantil y Educación Primaria, en la Facultad de Ciencias de la Educación de la Universidad de Córdoba (España) en el año académico 2011/12. Participaron en la investigación 274 estudiantes ( $81.4 \%$ mujeres), de los cuales 129 pertenecían a la especialidad de Educación Infantil (47.1\%) y 145 a la especialidad de Educación Primaria (52.9\%). La edad de los sujetos oscila entre los 18 y los 40 años $(\mathrm{M}=20.61$ años; D.T. $=$ 3.169). Se asumió un error muestral del $4 \%$ para un nivel de confianza del $97 \%$.

\section{Instrumentos}

Se utilizó el Maslach Burnout Inventory-Student Survey (MBI-SS: Schaufeli, Martínez et al., 2002; Schaufeli, Salanova et al., 2002), que evalúa el grado en que el estudiante está "quemado" por sus estudios. Este instrumento consta de tres dimensiones. La dimensión de agotamiento ( 5 ítems; $\alpha=.863$; ej.: "Estoy emocionalmente agotado por hacer esta carrera"), dimensión de cinismo (4 ítems; $\alpha=.790$; ej.: "He perdido interés en la carrera desde que empecé en la universidad") y dimensión de eficacia (6 ítems; $\alpha=.752$; ej.: "Creo que contribuyo efectivamente durante las clases en la universidad"). El conjunto de ítems que componen las dimensiones se presentan en una escala tipo likert con puntuaciones que van desde " 0 " (Ninguna vez/Nunca) a "6" (Todos los días/Siempre). 
También se utilizó un cuestionario compuesto por preguntas sobre: (a) datos sociodemográficos y académicos; (b) las competencias transversales o genéricas seleccionadas por la ANECA en el del Libro Blanco de la Titulación de Magisterio (ANECA, 2005), extraídas del Proyecto Tuning (González y Wagenaar, 2003); y (c) las competencias específicas del Libro Blanco de la Titulación de Magisterio comunes a todas sus especialidades (ANECA, 2005). Ambos listados de competencias se pueden consultar en la Figura 1 y Figura 2. Cada una de las competencias se sometía a la autovaloración del grado de desarrollo por parte del estudiante mediante una escala tipo likert de " 0 " (no se desarrolla) a " 6 " (se desarrolla mucho).

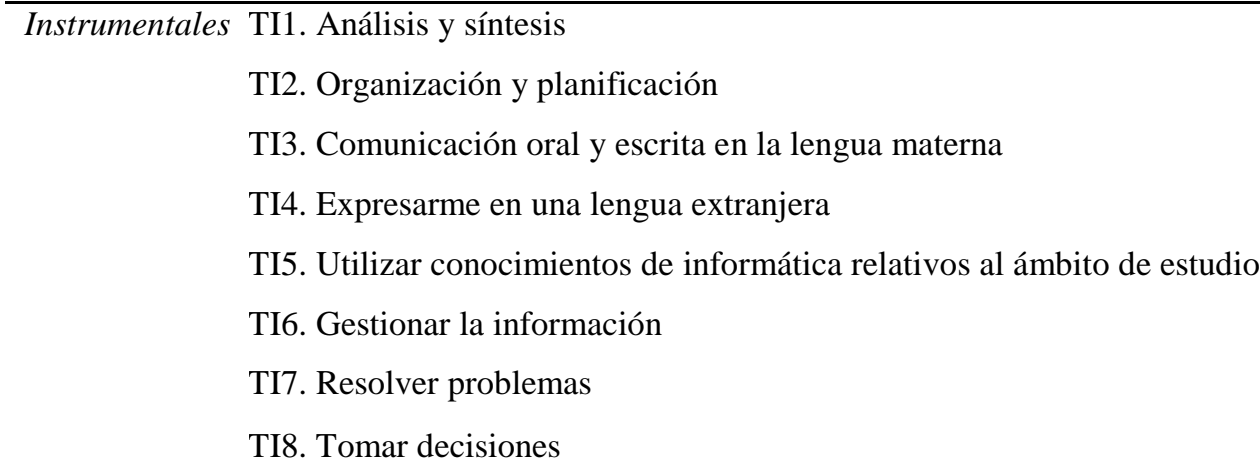

Figura 1. Competencias transversales 


\begin{tabular}{|c|c|}
\hline \multirow[t]{3}{*}{ Saber } & $\begin{array}{l}\text { ES1. Comprender la complejidad de los procesos educativos y de los procesos de enseñanza- } \\
\text { aprendizaje }\end{array}$ \\
\hline & ES2. Conocer los contenidos que hay que enseñar y cómo enseñarlos \\
\hline & ES3. Formación científico-cultural y tecnológica \\
\hline \multirow[t]{15}{*}{ Saber hacer } & $\begin{array}{l}\text { ESH4. Respetar las diferencias culturales y personales de los alumnos y demás miembros de la } \\
\text { comunidad educativa }\end{array}$ \\
\hline & ESH5. Analizar y cuestionar las propuestas curriculares de la Administración Educativa \\
\hline & $\begin{array}{l}\text { ESH6. Diseñar y desarrollar proyectos educativos y unidades de programación que permitan } \\
\text { adaptar el currículum al contexto sociocultural }\end{array}$ \\
\hline & ESH7. Promover el aprendizaje autónomo de los alumnos, desarrollando estrategias que eviten \\
\hline & la exclusión y la discriminación \\
\hline & $\begin{array}{l}\text { ESH8. Organizar la enseñanza utilizando de forma integrada los saberes disciplinares, trans- } \\
\text { versales y multidisciplinares adecuados al respectivo nivel educativo }\end{array}$ \\
\hline & $\begin{array}{l}\text { ESH9. Preparar, seleccionar o construir materiales didácticos y utilizarlos en los marcos es- } \\
\text { pecíficos de las distintas disciplinas }\end{array}$ \\
\hline & ESH10. Utilizar e incorporar adecuadamente en las actividades de enseñanza-aprendizaje las \\
\hline & tecnologías de la información y la comunicación \\
\hline & ESH11. Promover la calidad de los contextos en los que se desarrolla el proceso educativo \\
\hline & ESH12. Utilizar la evaluación como elemento regulador y promotor de la mejora de la ense- \\
\hline & ñanza, del aprendizaje y de su propia formación \\
\hline & ESH13. Realizar actividades educativas de apoyo en el marco de una educación inclusiva \\
\hline & $\begin{array}{l}\text { ESH14. Desempeñar la función tutorial, orientando a alumnos y padres y coordinando la } \\
\text { acción educativa referida a su grupo de alumnos }\end{array}$ \\
\hline & ESH15. Participar en proyectos de investigación relacionados con enseñanza- aprendizaje \\
\hline \multirow[t]{5}{*}{ Saber estar } & ESE16. Relacionarme, comunicarme y mantener el equilibrio emocional en todas situaciones \\
\hline & ESE17. Trabajar en equipo con los compañeros, compartiendo saberes y experiencias \\
\hline & $\begin{array}{l}\text { ESE18. Dinamizar con el alumnado la construcción participada de reglas de convivencia } \\
\text { democrática, y afrontar y resolver de forma colaborativa situaciones problemáticas y conflictos }\end{array}$ \\
\hline & interpersonales de naturaleza diversa \\
\hline & ESE19. Colaborar con los distintos sectores de la comunidad educativa y del entorno \\
\hline \multirow[t]{6}{*}{ Saber ser } & $\begin{array}{l}\text { ESS20. Tener una imagen realista de mí mismo, actuar conforme a las propias convicciones, } \\
\text { asumir responsabilidades, tomar decisiones y relativizar las posibles frustraciones }\end{array}$ \\
\hline & $\begin{array}{l}\text { ESS21. Asumir la dimensión ética del maestro potenciando en el alumnado una actitud de } \\
\text { ciudadanía crítica y responsable }\end{array}$ \\
\hline & ESS22. Potenciar el rendimiento académico de los alumnos y su progreso escolar, en el marco \\
\hline & de una educación integral \\
\hline & ESS23. Asumir la necesidad de desarrollo profesional continuo, mediante la autoevaluación de \\
\hline & la propia práctica \\
\hline
\end{tabular}

Figura 2. Competencias específicas 


\section{Procedimiento}

Los cuestionarios fueron distribuidos en noviembre de 2011, en horario lectivo con el consentimiento del profesorado. Los participantes, todos mayores de 18 años, fueron informados sobre el tratamiento confidencial de los datos que aportarían y posteriormente respondieron de forma anónima, individual y voluntaria. La duración de la realización del cuestionario fue en todos los casos entre 15-20 minutos.

\section{Análisis Estadístico}

Se utilizó el SPSS 15.0 para crear una matriz de datos y para realizar análisis estadísticos de corte descriptivo y comparativo. Sobre las percepciones de los estudiantes en el grado de desarrollo de sus competencias se estudiaron las medias y sus respectivas desviaciones típicas.

El análisis de conglomerado en dos fases nos permitió distribuir la muestra en grupos dependiendo del: (a) grado de desarrollo de competencias; y (b) nivel de burnout. Se utilizó el análisis de conglomerados para determinar la diferencia entre los "quemados" y "no quemados" debido a la inexistencia de puntuaciones que nos permitieran establecer puntos de corte para detectar indicios de burnout altos, medios o bajos. En ambos casos para la medida de la distancia se utilizó el criterio de máxima similitud y se optó por el criterio de conglomeración Bayesiano de Schwarz (BIC).

La prueba estadística $t$ de Students y ANOVA se utilizó para comprobar si existían diferencias entre las puntuaciones medias de diferentes grupos. Con respecto a la pruebas $t$ para muestras independientes utilizamos la prueba de Levene como criterio para asumir si las varianzas eran iguales o no. Los análisis post hoc que se realizaron, en el caso de las ANOVAS, para determinar entre qué grupos se hallaban las diferencias significativas se basaron en los estadísticos Tukey-b (cuando se asumieron varianzas iguales) y Games-Howell (cuando no se asumieron varianzas iguales). El nivel de confianza aplicado ha sido del 95\% $(p<.05)$ y del $99 \%(p<.01)$, dependiendo de los casos.

\section{Resultados}

\section{Diferencias en base al burnout}

El análisis cluster efectuado en dos fases, para determinar si existían diferentes grupos de estudiantes en función de las respuestas que dieron al MBI-SS (Schaufeli, Martínez et al., 
2002; Schaufeli, Salanova et al., 2002), separó la muestra en dos grupos (ver Figura 3), uno formado por 84 estudiantes que podían considerarse "quemados" (el 30.7\%), y otro formado por 190 estudiantes que denominamos "no quemados" (el 69.3\%). Denominamos así al primer grupo ya que presentaba puntuaciones superiores al segundo en las variables de agotamiento y cinismo y más bajas puntuaciones en eficacia, siendo todas estas diferencias estadísticamente significativas.

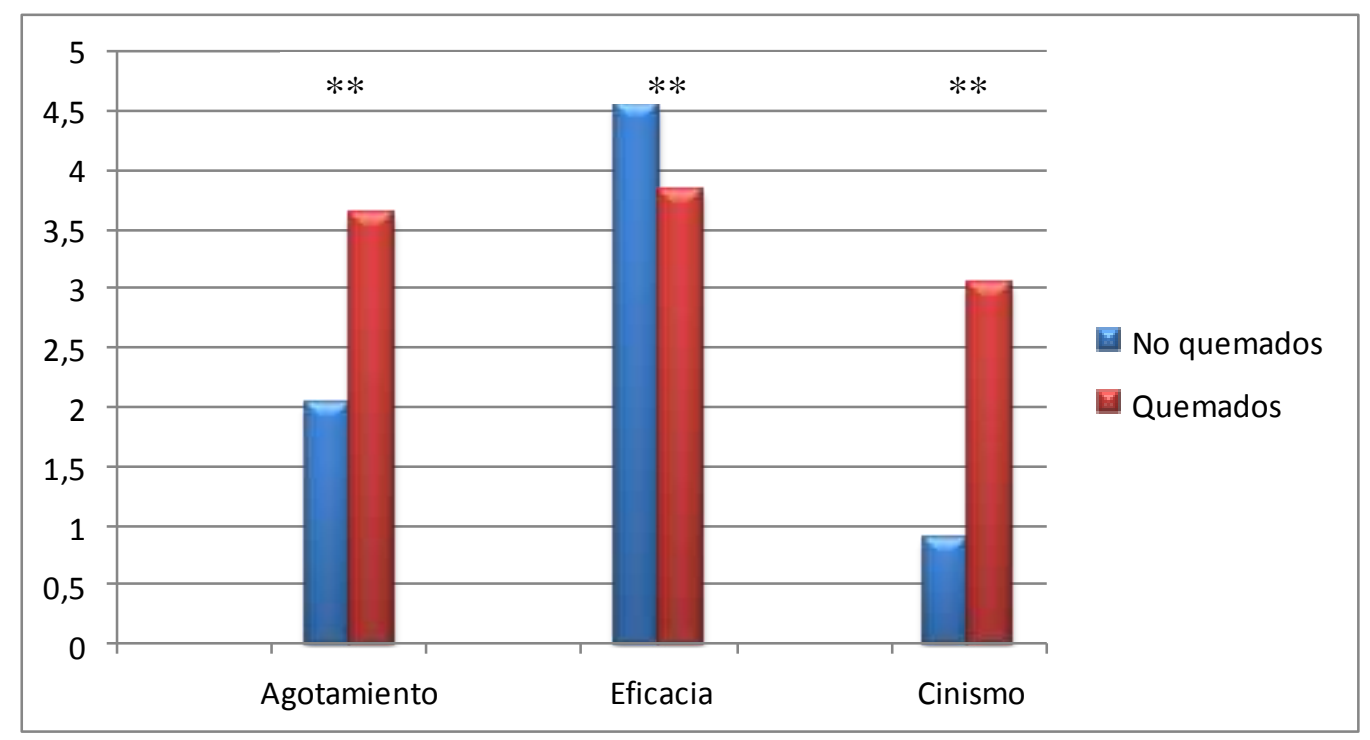

$* \mathrm{p}<.05 ; * * \mathrm{p}<.01$

Figura 3. Comparación entre las puntuaciones de "quemados" y "no quemados" en las dimensiones de burnout

\section{Diferencias en la percepción de competencias generales}

Respecto a la prueba $t$ para muestras independientes, para comparar si existían diferencias en el desarrollo estimado de competencias entre alumnado "quemado" y alumnado "no quemado", se encontraron diferencias significativas $(p<.05)$ entre ambos grupos en el desarrollo estimado de once competencias transversales (ver Tabla 1) y de seis competencias específicas (ver Tabla 2), siempre a favor de más desarrollo estimado de competencias en los "no quemados" respecto a los "quemados". 
Tabla 1. Diferencias significativas en la autopercepción del desarrollo de competencias transversales entre alumnos "quemados" y "no quemados".

\begin{tabular}{|c|c|c|c|c|c|c|c|c|c|c|}
\hline & \multirow[b]{3}{*}{ Grupo } & \multirow[b]{3}{*}{ Media } & \multirow[b]{3}{*}{ D. T. } & \multicolumn{7}{|c|}{ Prueba $t$ para la igualdad de medias } \\
\hline & & & & \multirow[t]{2}{*}{$\mathrm{t}$} & \multirow[t]{2}{*}{$\mathrm{gl}$} & \multirow[t]{2}{*}{$\mathrm{p}$} & \multirow{2}{*}{$\begin{array}{c}\text { Dif. } \\
\text { medias }\end{array}$} & \multirow{2}{*}{$\begin{array}{l}\text { Error típ. } \\
\text { de la dif. }\end{array}$} & \multicolumn{2}{|c|}{$\begin{array}{c}95 \% \text { Intervalo de } \\
\text { confianza para la diferencia }\end{array}$} \\
\hline & & & & & & & & & Inf. & Sup. \\
\hline \multirow{2}{*}{ TI2 } & No quemados & 4.57 & $(.991)$ & \multirow{2}{*}{2.835} & \multirow{2}{*}{266} & \multirow{2}{*}{.005} & \multirow{2}{*}{.375} & \multirow{2}{*}{.132} & \multirow{2}{*}{.115} & \multirow{2}{*}{.635} \\
\hline & Quemados & 4.20 & $(1.01)$ & & & & & & & \\
\hline \multirow{2}{*}{ TI5 } & No quemados & 4.37 & $(1.102)$ & \multirow{2}{*}{2.668} & \multirow{2}{*}{268} & \multirow{2}{*}{.008} & \multirow{2}{*}{.410} & \multirow{2}{*}{.154} & \multirow{2}{*}{.108} & \multirow{2}{*}{.713} \\
\hline & Quemados & 3.96 & $(1.301)$ & & & & & & & \\
\hline \multirow{2}{*}{ TP9 } & No quemados & 5.24 & $(.802)$ & \multirow{2}{*}{2.992} & \multirow{2}{*}{128.380} & \multirow{2}{*}{.003} & \multirow{2}{*}{.380} & 127 & 170 & 621 \\
\hline & Quemados & 4.86 & $(1.026)$ & & & & & .121 & .129 & . \\
\hline TP10 & No quemados & 4.48 & $(1.204)$ & & & 010 & 271 & 157 & & \\
\hline 1 PIO & Quemados & 4.11 & $(1.133)$ & 2.362 & 263 & .019 & $.3 / 1$ & .151 & .062 & .081 \\
\hline TP12 & No quemados & 5.23 & $(.919)$ & & 128271 & 014 & 260 & 148 & 070 & 651 \\
\hline $1 P 12$ & Quemados & 4.87 & $(1.177)$ & 2.490 & 128.271 & .014 & .362 & .140 & $.0 / 4$ & .051 \\
\hline ТР13 & No quemados & 5.15 & $(1.126)$ & 2185 & 267 & 020 & 200 & 147 & 022 & 600 \\
\hline 1110 & Quemados & 4.83 & $(1.063)$ & $2.10 \mathrm{~J}$ & $20 \%$ & .000 & $.3 \angle 0$ & $.14 /$ & .032 & (.009 \\
\hline ТР15 & No quemados & 4.91 & $(1.010)$ & 3002 & 265 & 003 & 410 & 1 & 144 & 604 \\
\hline 1815 & Quemados & 4.49 & $(1.152)$ & 3.002 & 203 & .003 & 419 & .140 & .144 & .094 \\
\hline TS17 & No quemados & 4.77 & $(1.016)$ & 40 & 260 & 015 & 325 & 17 & 063 & 588 \\
\hline דונו & Quemados & 4.45 & (1.003) & 2.440 & 209 & (01) & .525 & .153 & .003 & .500 \\
\hline TC? & No quemados & 4.52 & (1.158) & & & & & & & \\
\hline $1 \mathrm{~S} 21$ & Quemados & 4.18 & $(1.128)$ & 2.249 & 209 & .025 & .041 & .151 & .042 & . \\
\hline TC2? & No quemados & 4.53 & $(1.140)$ & 008 & 260 & 027 & 327 & 15 & 020 & 624 \\
\hline $1 \mathrm{~S} \angle 2$ & Quemados & 4.20 & $(1.217)$ & 2.098 & 209 & (201 & .522 & .153 & $.0 \angle 0$ & $.0 \angle 4$ \\
\hline S? & No quemados & 4.67 & $(1.174)$ & 052 & 268 & 050 & 337 & 16 & 003 & 636 \\
\hline 1523 & Quemados & 4.35 & $(1.337)$ & 1.952 & 208 & (050 & .317 & .102 & -.003 & .030 \\
\hline
\end{tabular}


Tabla 2. Diferencias significativas en la autopercepción del desarrollo de competencias específicas entre los alumnos "quemados" $y$ "no quemados".

\begin{tabular}{|c|c|c|c|c|c|c|c|c|c|c|}
\hline & \multirow[b]{3}{*}{ Grupo } & \multirow[b]{3}{*}{ Media } & \multirow[b]{3}{*}{ D. T. } & \multicolumn{7}{|c|}{ Prueba $t$ para la igualdad de medias } \\
\hline & & & & \multirow[t]{2}{*}{$\mathrm{t}$} & \multirow[t]{2}{*}{ gl } & \multirow[t]{2}{*}{$\mathrm{p}$} & \multirow{2}{*}{$\begin{array}{c}\text { Dif. } \\
\text { medias }\end{array}$} & \multirow{2}{*}{$\begin{array}{l}\text { Error típ. } \\
\text { de la dif. }\end{array}$} & \multicolumn{2}{|c|}{$\begin{array}{c}95 \% \text { Intervalo de } \\
\text { confianza para la diferencia }\end{array}$} \\
\hline & & & & & & & & & Inf. & Sup. \\
\hline \multirow{2}{*}{ ES1 } & No quemados & 4.57 & $(.890)$ & \multirow{2}{*}{-2.073} & \multirow{2}{*}{269} & \multirow{2}{*}{.039} & \multirow{2}{*}{.244} & \multirow{2}{*}{.118} & \multirow{2}{*}{.012} & \multirow{2}{*}{.475} \\
\hline & Quemados & 4.33 & $(.899)$ & & & & & & & \\
\hline \multirow{2}{*}{ ESH4 } & No quemados & 5.35 & $(.855)$ & \multirow{2}{*}{-3.648} & \multirow{2}{*}{116.290} & \multirow{2}{*}{.000} & \multirow{2}{*}{.556} & \multirow{2}{*}{.152} & \multirow{2}{*}{.254} & \multirow{2}{*}{.858} \\
\hline & Quemados & 4.80 & $(1.266)$ & & & & & & & \\
\hline \multirow{2}{*}{ ESE16 } & No quemados & 4.71 & $(1.071)$ & \multirow{2}{*}{2.244} & \multirow{2}{*}{133.899} & \multirow{2}{*}{.026} & \multirow{2}{*}{.363} & \multirow{2}{*}{.162} & \multirow{2}{*}{.043} & \multirow{2}{*}{.684} \\
\hline & Quemados & 4.35 & $(1.292)$ & & & & & & & \\
\hline \multirow{2}{*}{ ESE17 } & No quemados & 5.08 & $(.970)$ & \multirow{2}{*}{2.323} & 127050 & (027 & 357 & 154 & 053 & 661 \\
\hline & Quemados & 4.72 & $(1.243)$ & & 121.950 & .022 & ו & .104 & (0J3 & (.001 \\
\hline ECE 10 & No quemados & 4.35 & $(1.297)$ & 2177 & 268 & 030 & 358 & 164 & 034 & 682 \\
\hline LSLIV & Quemados & 3.99 & $(1.105)$ & 2.171 & 208 & (050 & .358 & .104 & .034 & . \\
\hline ECC & No quemados & 4.74 & $(1.039)$ & 781 & 178056 & 006 & 152 & $16 ?$ & 131 & 773 \\
\hline ESS 20 & Quemados & 4.29 & $(1.300)$ & 2.184 & 120.050 & 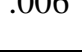 & .452 & .102 & .151 & .113 \\
\hline
\end{tabular}

\section{Diferencias en la percepción de competencias transversales y específicas}

Las puntuaciones obtenidas en las competencias transversales permitieron clasificar al alumnado en dos grupos diferenciados a través del análisis de conglomerados en dos fases. Un primer grupo de sujetos que presentan una autopercepción del nivel de desarrollo de competencias más elevado, formado por 128 sujetos (el 49.6\%); y un segundo grupo cuya percepción sobre sus competencias es sensiblemente inferior al primer grupo, compuesto por 130 sujetos (50.4\%). Respecto a la autopercepción del desarrollo de competencias específicas expresada en puntuaciones, también se clasificó al alumnado mediante otro análisis de conglomerados en dos fases, resultando tres grupos. Un primer grupo, de nivel elevado, compuesto por 126 sujetos (el 47.7\%); un segundo grupo, de nivel medio, formado por 104 sujetos (el $39.4 \%$ ); y un tercer grupo, de nivel inferior, compuesto por 34 sujetos (el 12.9\%).

Se realizaron pruebas $t$ para muestras independientes para comprobar si existían diferencias entre el grupo que estima más desarrolladas y el grupo que estima menos desarrolladas sus competencias transversales, respecto a las dimensiones del síndrome de burnout. Se encontraron diferencias estadísticamente significativas únicamente en la dimensión de efica- 
cia $\left(t_{258}=3.331, p<.01\right)$ y no se detectaron ni en agotamiento $\left(t_{258}=-1.795, p>.05\right)$ ni en cinismo $\left(t_{258}=-1.311, p>.05\right)$.

Respecto a la autopercepción de desarrollo de competencias específicas, clasificada en tres niveles (elevado, medio y bajo) se realizó un ANOVA para comprobar las posibles diferencias entre los tres grupos establecidos en relación a las dimensiones del burnout. Se encontró también significación estadística únicamente en la dimensión de eficacia $[F(2,261)=$ $3.402, p<.05]$. Las pruebas pos-hoc no detectaron entre qué grupos se encontraban las diferencias.

\section{Discusión y conclusiones}

Como se esperaba, el alto nivel de burnout académico mostraba una clara relación con niveles más bajos de auto-percepción en el desarrollo de competencias. En el contraste entre grupos de estudiantes con nivel alto y bajo/nulo de burnout respecto al grado de desarrollo de competencias, siempre que se registraron diferencias significativas fueron negativas en relación al grupo con alto burnout. Así se evidenció en once de las veintitrés competencias genéricas o transversales, y en seis de las veintitrés competencias específicas.

En dos de las tres dimensiones del burnout académico -agotamiento y cinismo- no se han observado diferencias significativas al comparar los niveles (alto vs. bajo) de desarrollo de las competencias transversales y específicas. Sin embargo, sí se han registrado diferencias significativas en relación a la dimensión que es considerada por algunos investigadores como el factor principal del burnout (Martínez y Salanova, 2003; Salanova, Cifre, et al., 2005) o un predictor importante del mismo (Schwarzer y Hallum, 2008): la eficacia. Se ha observado una vinculación positiva entre los niveles auto-estimados de competencias y de eficacia, hecho concordante con las conclusiones de algunos estudios previos (p. ej.: Salanova, Bresó et al., 2005).

Si bien los resultados del presente estudio, debido a su diseño, no permiten describir el sentido de la relación detectada entre desarrollo competencial y burnout académico -si el primero produce el segundo o viceversa- no son discordantes con los expuestos en los estudios de Hultell et al. (2013), Bresó et al. (2011) y Caballero et al. (2010). Estos estudios concluyen, apoyados sobre la Teoría Socio-Cognitiva, que la crisis de la competencia puede tener 
un impacto negativo en la auto-percepción de la eficacia del propio sujeto, originando el desarrollo del burnout. Considerando el sentido descrito de ésta relación, que para algunos investigadores la crisis de la eficacia está identificada indisolublemente con el burnout (GarcíaRenedo, Llorens, Cifre y Salanova, 2006; Salanova, Cifre et al., 2005) y que la autoeficacia conduce al éxito académico (García-Ros y Pérez-González, 2011; Salanova et al., 2012), pensamos que para revertir y prevenir el burnout y mejorar el rendimiento académico de estudiantes podría ser especialmente efectivo el desarrollo de estrategias orientadas a producir un impacto positivo en la autoeficacia del aprendiz. Estas estrategias podrían ser de tipo terapéutico y/o educativo.

\section{Implicaciones para la intervención}

Entre las posibles estrategias de tipo terapéutico en los últimos años se han recogido evidencias de la eficacia de los programas de intervención cognitivo-conductual. Por ejemplo, el estudio de Bresó et al. (2011) muestra resultados positivos en la reducción de la ansiedad y en el aumento de la autoeficacia, por la acción de un terapeuta orientada al desarrollo de competencias emocionales del alumnado universitario, la reducción de sus sentimientos de incompetencia y su ansiedad al enfrentarse a los exámenes. Para ello el programa se centró en el trabajo sobre la intolerancia a la incertidumbre, las creencias erróneas sobre las preocupaciones, la falta de orientación hacia los problemas y la evitación cognitiva de las y los estudiantes.

Las estrategias de tipo educativo para revertir o prevenir el burnout y mejorar el rendimiento académico, estarían centradas tanto en la mejora del desarrollo competencial del aprendiz como en la mejora de su auto-percepción de dichas competencias. Sin embargo ésta inferencia nos conduce a una nueva cuestión: habría que saber sobre qué tipos de competencias debería centrarse este trabajo. El presente estudio puede contribuir con sus resultados a avanzar en la respuesta a esta pregunta, especialmente respecto a estudiantes del Grado de Maestro/a.

La relación entre bajo desarrollo competencial y mayor nivel de burnout, dentro de las competencias transversales, se observó más en las competencias de tipo personal (en cinco de siete: TP9, TP10, TP12, TP13 y TP15) y sistémicas (en cuatro de ocho: TS17, TS21, TS22 y TS23) que en las instrumentales (en dos de ocho: TI2 y TI5). El burnout parecer así tener mayor relación con la autoevaluación negativa en cuanto a aptitudes y actitudes de subtipos: (a) intrapersonal, como el compromiso ético o reconocer la diversidad y la multiculturalidad; y 
(b) interpersonal, como relacionarse con otras personas o trabajar en equipo (disciplinar o interdisciplinar). También se observa que el burnout tiene mayor relación con el bajo nivel de competencias ligadas a la relación del sujeto con el sistema/contexto, tales como la adaptación a nuevas situaciones, la toma de la iniciativa y el emprendimiento, o la promoción de la calidad.

La relación entre bajo desarrollo de competencias específicas de maestros/as y mayor nivel de burnout volvió a mostrarse más en competencias que podrían considerarse relacionadas con lo personal y lo sistémico/contextual. Las competencias específicas que parecen ligadas a lo personal son: (a) intrapersonales, como tener una imagen realista de mí mismo, actuar conforme a las propias convicciones, asumir responsabilidades, tomar decisiones y relativizar las posibles frustraciones; y (b) interpersonales, como relacionarse comunicarse y mantener el equilibrio emocional, o trabajar en equipo con los compañeros, compartiendo saberes y experiencias. Las competencias específicas que parecen guardar relación con lo sistémico/contextual son: (a) comprender la complejidad de los procesos educativos y de los procesos de enseñanza-aprendizaje; (b) respetar las diferencias culturales y personales de los alumnos y demás miembros de la comunidad educativa; (c) y colaborar con los distintos sectores de la comunidad educativa y del entorno. A través de los resultados obtenidos en sus estudios, Pérez-Escoda et al. (2012) apoyan la idea de que los sistemas educativos deberían incorporar programas destinados al desarrollo de competencias emocionales, fomentando así el bienestar personal y social de alumnado.

Algunas recientes investigaciones muestran que casi la mitad de los estudiantes evidencian problemas de competencia en habilidades interpersonales (Shen-Miller et al., 2012) y que particularmente algunos estudiantes de magisterio no cuentan con las habilidades suficientes para trabajar en equipo, en el manejo de personas, en adaptarse a los continuos cambios o controlar sus emociones (Pertegal-Felices et al., 2011). Por su parte, Castejón et al. (2008) menciona la necesidad de favorecer el desarrollo de la inteligencia interpersonal en universitarios a través de métodos de enseñanza que estimulen el trabajo cooperativo y dinámicas de grupos, entre otros. Estas evidencias junto con las detectadas en el presente estudio nos conducen a plantear la necesidad de abordar un trabajo formativo y de asesoramiento focalizado en la estimulación y desarrollo de competencias de tipo personal (intrapersonales e interpersonales) y sistémico/contextual sobre estudiantes de magisterio para mejorar su autoeficacia y poder prevenir el burnout y mejorar sus rendimiento académico. Al igual que en 
conclusiones obtenidas por Palomera et al. (2008), pensamos que parece prioritario centrar mayor esfuerzo formativo en el desarrollo de este tipo de competencias, en contraste con los tipos de competencias que tradicionalmente se vienen trabajando bien en la formación superior de educadores, como son las instrumentales y de corte más conceptual y procedimental. A demás, el reconocimiento y comprensión de los estados emocionales de uno mismo y de los demás, resulta favorable para el desarrollo de recursos y estrategias de afrontamiento a situaciones estresantes (Gustems y Calderón, 2014).

\section{Limitaciones y prospectivas}

La muestra, siendo representativa de la población descrita, no permite generalizar a otras poblaciones los resultados. El instrumento para medir las competencias, diseñado ad hoc, no mide variables a través de escalas, lo que dificulta análisis más avanzados. Del mismo modo no mide un constructo, sino una serie de ítems aislados. El diseño del estudio, dado su carácter transversal, no nos permite predecir si los valores de burnout son los causantes de la autopercepción del desarrollo de competencia o viceversa. Aun así, el estudio nos ha aportado datos valiosos para seguir avanzando en el conocimiento y futuro estudio en la materia. Para próximas investigaciones pretendemos aumentar la muestra y representatividad de las poblaciones de referencia, ampliando el rango a otras titulaciones universitarias. Sería de interés incluir mediciones del engagement, concepto opuesto del burnout, para comprobar si existe relación entre éste y el desarrollo de competencias.

\section{Agradecimientos}

El presente trabajo es parte del I Proyecto Interfacultades de Innovación en Prácticas Externas Curriculares: Desarrollo de Competencias Profesionales, código: 2013-11-6001, subvencionado y concedido por la Universidad de Córdoba a Antonio J. Rodríguez-Hidalgo (IP) para su desarrollo en tres centros: Facultad de Ciencias de la Educación, Facultad de Veterinaria y Facultad de Filosofía y Letras. 


\section{Referencias}

Agencia Nacional de Evaluación de la Calidad y Acreditación (ANECA). (2005). Libro Blanco del Título de Grado en Magisterio. Vol.1. Recuperado de http://www.aneca.es/Documentos-y-publicaciones/Libros-Blancos

Arís, N. (2009). Burnout Syndrome in educators. Electronic Journal of Research in Educational Psychology, 7(2), 829-848. Recuperado de http://www.investigacionpsicopedagogica.org/revista/new/english/ContadorArticulo.php?303

Bandura, A. (1997). Self-efficacy. The exercise of control. New York: Freeman.

Bandura, A. (2012). On the Functional Properties of Perceived Self-Efficacy Revisited. Journal of Management, 38(1), 9-44. doi: 10.1177/0149206311410606.

Beck, J. W. y Schmidt, A. M. (2012). Taken out of context? Cross-level effects of betweenperson self-efficacy and difficulty on the within-person relationship of self-efficacy with resource allocation and performance. Organizational Behavior and Human Decision Processes, 119, 195-208. doi:10.1016/j.obhdp.2012.06.009

Beneitone, P. Esquetini, C., González, J., Marty, M., Siufi, G. Wagenaar, R. (2007). Reflexiones y perspectivas de la Educación Superior en América Latina, Informe Final Proyecto Tuning-América Latina 2004-2007. Universidad de Deusto y Universidad Groningen.

Bresó, E., Salanova, M. y Schaufeli, W. (2007). In Search of the "Third Dimension” of Burnout: Efficacy or Inefficacy? Applied Psychology: An International Review, 56(3), 460478. doi: 10.1111/j.1464-0597.2007.00290.x.

Bresó, E., Schaufeli, W. B. y Salanova, M. (2011). Can a self-efficacy-based intervention decrease burnout, increase engagement, and enhance performance? A quasiexperimental study. High Educ, 61, 339-355. doi 10.1007/s10734-010-9334-6.

Caballero, C., Hederich, C. y Palacio, J. (2010). El burnout académico: delimitación del síndrome y factores asociados con su aparición. Revista Latinoamericana de Psicología, 42(1), 131-146. $\quad$ Recuperado de http://www.redalyc.org/pdf/805/80515880012.pdf

Castejón J. L., Cantero, M. P. y Pérez, N. (2008). Diferencias en el perfil de competencias socio-emocionales en estudiantes universitarios de diferentes ámbitos científicos. Electronic Journal of Research in Educational Psychology, 15(6), 339-362. Recuperado de http://www.investigacionpsicopedagogica.org/revista/new/ContadorArticulo.php?267

Cho, Y. y Serena, S. (2013). Predicting teachers' achievement goals for teaching: The role of perceived school goal structure and teachers' sense of efficacy. Teaching and Teacher Education, 32, 12-21. doi:10.1016/j.tate.2012.12.003 
de la Fuente, J., Justicia, F., Casanova, P. F. y Trianes, M. V. (2005). Percepción sobre la construcción de competencias académicas y profesionales en Psicólogos. Electronic Journal of Research in Educational Psychology, 5-3(1), 3-34. Recuperado de http://www.investigacion-psicopedagogica.org/revista/new/ContadorArticulo.php?57

Durán, A., Extremera, N., Rey, L., Fernández-Berrocal, P. y Montalbán, F. M. (2006). Predicting academic burnout and engagement in educational settings: Assessing the incremental validity of perceived emotional intelligence beyond perceived stress and general self-efficacy. Psicothema, 18, 158-164. Recuperado de http://www.psicothema.com/pdf/3292.pdf

Fontes de Oliva, E., Andrade S., Rodrigues de Abreu, A. T., Vieira, E. y Matos, T. (2012). Burnout Syndrome and associated factors among medical students: a cross-sectional study. Clinics, 67(6), 573-579. doi: 10.6061/clinics/2012(06)05.

García-Renedo, M., Llorens, S., Cifre, E. y Salanova, M. (2006). Antecedentes afectivos de la auto-eficacia docente: un modelo de relaciones estructurales. Revista de Educación, 339, 387-400. Recuperado de http://www.revistaeducacion.mec.es/re339/re339_17.pdf

García-Ros, R. y Pérez-González, F. (2011). Validez predictiva e incremental de las habilidades de autorregulación sobre el éxito académico en la universidad. Revista de Psicodidáctica, 16(2), 231-250. doi: 10.1387/RevPsicodidact.1002

Gil-Monte, P.R. y Peiró, J.M. (1999). Validez factorial del Maslach Burnout Inventory en una muestra multiocupacional. Psicothema, 11(3), 679-689. Recuperado de http://www.psicothema.com/pdf/319.pdf

González, A., Donolo, D., Rinaudo, C. y Paoloni, V. (2011). Relaciones entre motivación, emoción y rendimiento académico en universitarios. Estudios de Psicología, 32 (2), 257-270.

González, J. y Wagenaar, R. (2003). Tuning educational Structures in Europe. Final Report. Phase one. Bilbao: Servicio de Publicaciones de la Universidad de Deusto.

González, M. T. y. Landero, R. (2007). Escala de cansancio emocional (ECE) para estudiantes universitarios: Propiedades psicométricas en una muestra de México. Anales de psicología, 23(2), 253-257. Recuperado de http://revistas.um.es/analesps/article/view/22321/21601

Gustems, J. y Calderón, C. (2014). Empathy and coping strategies as predictors of well-being in Spanish university students. Electronic Journal of Research in Educational Psychology, 12(1), 129-146. doi: 10.14204/ejrep.32.13117

Hultell, D., Melin, B. y Gustavsson, J. P. (2013). Getting personal with teacher burnout: A longitudinal study on the development of burnout using a person-based approach. Teaching and Teacher Education 32, 75-86. doi:10.1016/j.tate.2013.01.007 
Malinen, O. P., Savolainen, H., Engelbrecht, P., Xu, J., Nel, M., Nel, N., y Tlale, D. (2013). Exploring teacher self-efficacy for inclusive practices in three diverse countries. Teaching and Teacher Education, 33, 34-44. doi:10.1016/j.tate.2013.02.004

Manzano, G. (2002). Burnout y engagement. Relación con el desempeño, madurez profesional y tendencia al abandono de los estudiantes. Revista de Psicología Social, 17(3), 237-249. doi:10.1174/02134740260372973

Martínez, I. y Salanova, M. (2003). Niveles de burnout y engagement en estudiantes universitarios. Relación con el desempeño y desarrollo profesional. Revista de Educación, 330, 361-384. Recuperado de http://www.mecd.gob.es/dctm/revista-deeducacion/articulosre330/re3301911213.pdf?documentId=0901e72b81258cd 9

Maslach, C. y Jackson, S. E. (1981). The measurement of experienced burnout. Journal of Occupational Behaviour, 2, 99-113. doi: 10.1002/job.4030020205

Palmer. A., Montaño, J. y Palou, M. (2009). Las competencias genéricas en la educación superior. Estudio comparativo entre la opinión de empleadores y académicos. Psicothema, 21(3), 433-438. Recuperado de http://www.psicothema.com/pdf/3650.pdf

Palomera, R., Fernández-Berrocal, P. y Brackett, M. A. (2008). La inteligencia emocional como una competencia básica en la formación inicial de los docentes: algunas evidencias. Electronic Journal of Research in Educational Psychology, 15(6), 437-454. Recuperado de http://www.redalyc.org/articulo.oa?id=293121924010

Pena, M., Rey, L., y Extremera, N. (2012). Life Satisfaction and Engagement in Elementary and Primary Educators: Differences in Emotional Intelligence and Gender. Revista de Psicodidáctica, 17(2), 341-358. doi: 10.1387/Rev.Psicodidact.1220.

Pérez-Escoda, N., Filella, G., Alegre, A. y Bisquerra, R. (2012). Developing the emotional competence of teachers and pupils in school context. Electronic Journal of Research in Educational Psychology, 10(3), 1183-1208. Recuperado de http://www.investigacionpsicopedagogica.org/revista/new/english/ContadorArticulo.php?756

Pertegal-Felices, M. L., Castejón-Costa, J. y Martínez, M. A (2011). Competencias socioemocionales en el desarrollo profesional del maestro. Educación XX1, 14(2), 237-260. doi: 10.5944/educxx 1.14.2.253

Sáiz, M. C. y Román, J. M. (2011). Cuatro formas de evaluación en educación superior gestionadas desde la tutoría. Revista de Psicodidáctica, 16(1), 145-161. doi: 10.1387/RevPsicodidact.1116

Salanova, M., Bresó, E. y Schaufeli, W. (2005). Hacia un modelo espiral de las creencias de eficacia en el estudio del burnout y del engagement. Ansiedad y Estrés, 11(2-3), 215231. Recuperado de http://www.wilmarschaufeli.nl/publications/Schaufeli/243.pdf 
Salanova, M., Cifre, E., Grau, R., Llorens, S. y Martínez, I. (2005). Antecedentes de la autoeficacia en profesores y estudiantes universitarios: un modelo casual. Revista de Psicología del Trabajo y de las Organizaciones, 21(1-2), 159-176. Recuperado de http://www.redalyc.org/pdf/2313/231317039010.pdf

Salanova, M., Martínez, I. y Llorens, S. (2012). Success breeds success, especially when selfefficacy is related with an internal attribution of causality. Estudios de Psicología, $33(2), 151-165$.

Salanova, M., Martínez, I., Bresó, E., Llorens, S. y Grau, R. (2005). Bienestar psicológico en estudiantes universitarios: facilitadores y obstaculizadores del desempeño académico. Anales de psicología, 21(1), 170-180. Recuperado de http://www.um.es/analesps/v21/v21_1/16-21_1.pdf

Schaufeli, W. B., Leiter, M. P., Maslach, C. y Jackson, S. E. (1996). Maslach Burnout Inventory-General Survey. En C. Maslach; S. E. Jackson; M. P. Leiter (Eds.): The Maslach Burnout Inventory-Test Manual ( $3^{\text {rd }}$ ed.). Palo Alto, CA: Consulting Psychologists Press.

Schaufeli, W., Martínez, I., Marques, A., Salanova, M. y Bakker, A. (2002). Burnout and engagement in university students: a cross national study. Journal of Cross-Cultural Psychology, 33(5), 464-481. doi: 10.1177/0022022102033005003

Schaufeli, W., Salanova, M., González-Romá, V. y Bakker, A. (2002). The measurement of engagement and burnout: a two simple confirmatory factor analytic approach. Journal of Happiness Studies, 3, 71-92. doi: 10.1023/A:1015630930326

Schwarzer, R. y Hallum, S. (2008). Perceived Teacher Self-Efficacy as a Predictor of Job Stress and Burnout: Mediation Analyses. Applied psychology: an international review, 57, 152-171. doi: 10.1111/j.1464-0597.2008.00359.x.

Shen-Miller, D. S., Grus, C. L.; Van Sickle, K. S., Schwartz-Mette, R., Cage, E. A., Elman, N. S., ... y Kaslow, N. J. (2011). Trainees' experiences with peers having Competence problems: A national survey. Training and Education in Professional Psychology, 5(2), 112-121. doi: 10.1037/a0023824.

Valdivieso, J. A., Carbonero, M. A., y Martín-Antón, L. J. (2013). Elementary School Teachers' Self-perceived intructional competence: a new questionnaire. Revista de Psicodidáctica, 18(1), 47-80. doi: 10.1387/RevPsicodidact.5622.

Villardón-Gallego, L., Yániz, C., Achurra, C., Iraurgi I., y Aguilar, C. (2013). Learning competence in university: development and structural validation of a scale to measure. $R e$ vista de Psicodidáctica, 18(2), 357-374. doi: 10.1387/RevPsicodidact.6470. 